\title{
Bioactive flavonoid $p$-hydroxycinnamic acid stimulates osteoblastogenesis and suppresses adipogenesis in bone marrow culture
}

\author{
Masayoshi Yamaguchi • Clifton A. Baile • Shijun Zhu • \\ Mamoru Shoji
}

Received: 5 June 2013 / Accepted: 16 July 2013 / Published online: 13 September 2013

(C) The Author(s) 2013. This article is published with open access at Springerlink.com

\begin{abstract}
The bioactive flavonoid $p$-hydroxycinnamic acid (HCA), which is an intermediate-metabolic substance in plants and fruits, is synthesized from tyrosine. The biological effect of HCA is poorly understood. Among cinnamic acid and its related compounds, HCA has a specific-anabolic effect on bone, being found to stimulate osteoblastogenesis and to inhibit osteoclastogenesis through the suppression of NF-KB signaling, thereby preventing bone loss. Bone marrow mesenchymal stem cells give rise to ostoblasts and adipocytes. HCA might therefore have effects on osteoblastogenesis and adipogenesis in bone marrow culture. This study demonstrates (1) that HCA has stimulatory effects on osteoblastogenesis and mineralization and suppressive effects on adipogenesis in mouse bone marrow culture and (2) that HCA depresses adipogenesis in mouse 3T3-L1 preadipocytes in vitro. Such effects of HCA might be involved in the differentiation of mesenchymal stem cells.
\end{abstract}

The authors designed and conducted the study. They also carried out the collection, analysis and interpretation of the data and wrote the manuscript. They declare no conflicts of interest.

M. Yamaguchi $\cdot$ S. Zhu $\cdot$ M. Shoji

Department of Hematology and Biomedical Oncology,

Winship Cancer Institute, Emory University School of Medicine,

Atlanta, GA 30322, USA

C. A. Baile

Department of Foods and Nutrition, The University of Georgia, Athens GA 30602, USA

M. Yamaguchi $(\square)$

Department of Hematology and Medical Oncology,

Winship Cancer Institute, Emory University School of Medicine,

1365 C Clifton Road NE, Atlanta, GA 30322, USA

e-mail: yamamasa1155@yahoo.co.jp
Keywords $p$-Hydroxycinnamic acid $\cdot$ Osteoblastogenesis . Adipogenesis $\cdot 3 \mathrm{~T} 3$-L1 preadipocytes $\cdot$ Insulin

\section{Introduction}

Bone homeostasis is maintained through a delicate balance between osteoblastic bone formation and osteoclastic bone resorption. Numerous pathological processes have the capacity to disrupt this equilibrium leading to conditions in which the rate of bone resorption outpaces the rate of bone formation leading to osteoporosis (Weitzmann and Pacifici 2006). The most dramatic expression of osteoporosis is represented by fractures of the proximal femur for which the number increases as the population ages (Johnell and Kanis 2006). Osteoporosis is widely recognized as a major public health threat.

Obesity is currently a major health problem worldwide and is growing in prevalence. Osteoporosis and obesity are now thought to be closely related and to share several features (Gharibi et al. 2011; Rosen and Bouxsein 2006; Kawai and Rosen 2010). One of these shared features is that osteoblasts and adipocytes differentiate from a common precursor cell in the bone marrow, the mesenchymal stem cell. The pluripotency of mesenchymal stem cells is well known and their ability to differentiate into osteoblasts, adipocytes, chondrocytes and myoblasts has been described extensively (Minguell et al. 2001). An inverse relationship exists between the differentiation of mesenchymal stem cells into osteoblasts and adipocytes. Secondary causes of osteoporosis including obesity and diabetes are associated with bone marrow adiposity (Gharibi et al. 2011).

Functional food factors might be important in the prevention and treatment of osteoporosis and obesity. Botanical chemical factors that have an effect on osteoblastogenesis and adipogenesis are poorly understood. Cinnamic acid, a 
flavonoid, is present in many plants and fruits. The flavonoid $p$-hydroxycinnamic acid (HCA) is an intermediate-metabolic substance in plants and fruits and is synthesized from tyrosine. Among cinnamic acid and its related compounds (cinnamic acid, HCA, ferulic acid, caffeic acid and 3,4-dimethoxycinnamic acid), HCA has been shown to have specific-anabolic effects on bone in vitro (Lai and Yamaguchi 2006a). HCA has been found to stimulate osteoblastic bone formation and to inhibit osteoclastic bone resorption in vitro (Lai and Yamaguchi 2007a; Yamaguchi et al. 2008a) and in vivo (Lai and Yamaguchi 2006b, 2007b; Yamaguchi et al. 2008b), thereby increasing bone mass. HCA has also been found to stimulate osteoblastogenesis and to inhibit osteoclastogenesis through the suppressing of nuclear factor kappa B (NF- $\mathrm{kB}$ ) signaling, which is activated by tumor necrosis factor-alpha (TNF- $\alpha)$ or the receptor activator of NF-KB ligand (RANKL; Yamaguchi and Weitzmann 2009, 2012), suggesting a molecular mechanism by which HCA has an anabolic effect on bone.

Moreover, HCA has been demonstrated to have preventive effects on ovariectomy-induced bone loss in rats (Yamaguchi et al. 2008b) and the compound has restorative effects on the diabetic state and diabetic-induced bone loss in streptozotocininduced type I diabetic rats in vivo (Lai and Yamaguchi 2007b). Oral administration of HCA in streptozotocininduced diabetic rats has also been shown to reduce serum triglyceride and glucose concentrations, which are markedly elevated in the diabetic state in vivo, suggesting that HCA has an effect on lipid metabolism (Lai and Yamaguchi 2007b).

Bone marrow mesenchymal stem cells are multipotent cells, which among other cell lineages, give rise to adipocytes and osteoblasts (Muruganandan et al. 2009). This occurs through cross talk between complex signaling pathways including those derived from bone morphogenic proteins, winglesstype MMTV integration site (Wnt) proteins, the hedgehog proteins, delta/jagged proteins, fibroblastic growth factors, insulin, insulin-like growth factors and transcriptional regulators of adipocyte and osteoblast differentiation including peroxisome proliferators-activated receptor-gamma (PPAR $\gamma$ ) and runt-related transcription factor 2 (Runx2; Muruganandan et al. 2009; Laudes 2011). Recently, dietary-induced serum phenolic acids including HCA have been shown to promote bone growth via p38 mitogen-activated protein kinase (MAPK)/beta-catenin canonical Wnt (Chen et al. 2010).

HCA might have effects on the differentiation of bone marrow mesenchymal stem cells and adipogenesis, which is related to differentiation of bone marrow mesenchymal stem cells to adipocytes. The effects of HCA on osteoblastogenesis and adipogenesis in bone marrow culture have not as yet been determined. This study has been undertaken to determine the effects of HCA on osteoblastogenesis and adipogenesis in mouse bone marrow culture and on adipogenesis in mouse 3T3-L1 preadipocytes in vitro. We have found that HCA has stimulatory effects on osteoblastogenesis and suppressive effects on adipogenesis.

\section{Materials and methods}

Materials

Dulbecco's modified Eagle's medium (DMEM) and antibiotics (penicillin and streptomycin) were purchased from Invitrogen (Carlsbad, Calif., USA). Fetal bovine serum (FBS) was from Hyclone. HCA (100 \% pure) was obtained from Wako Pure Chemical (Osaka, Japan) and was dissolved in ethanol for use in experiments. Insulin, dexamethasone, 3isobutyl-1-methylxanthine (IBMX), PD98059, staurosporine and other reagents were purchased from Sigma (St. Louis, Mo., USA). Insulin was dissolved in diluted acidic acid solution and other reagents were dissolved in $100 \%$ ethanol.

Mineralization in mouse bone marrow culture

Bone marrow cells $\left(1 \times 10^{6}\right.$ cells/wells per $\left.1 \mathrm{ml}\right)$, which were isolated from mice (C57BL6 wild-type; female, 2 months old) purchased from the Jackson Laboratory (USA), were cultured in 12-well plates in DMEM containing $10 \%$ FBS, $1 \%$ penicillin-streptomycin $(\mathrm{P} / \mathrm{S} ; 10,000 \mathrm{U} / \mathrm{l})$ and mineralization medium (MM) containing ascorbic acid $(100 \mathrm{ng} / \mathrm{ml})$ and $4 \mathrm{mM} \beta$-glycerophosphate in the presence of either vehicle or HCA (1-1000 nM; Yamaguchi et al. 2012). Cells were cultured for 21 days at $37{ }^{\circ} \mathrm{C}$ in a humidified $5 \% \mathrm{CO}_{2}$ atmosphere. In other experiments, the cells were cultured for 7 days in the presence of HCA $(1-1000 \mathrm{nM})$ or its absence, after which the medium was replaced by medium without HCA and the cells were cultured for an additional 14 days. The medium was changed every 3 days. After culture, the cells were washed with phosphate-buffered saline and stained with Alizarin red stain. For quantitation, $10 \%$ cetylpyridinium chloride solution was added to each well to elute the dye. After complete elution, absorbance of the eluted solution was measured at $570 \mathrm{~nm}$ on a microtiter plate reader.

Adipogenesis in bone marrow culture

Bone marrow cells $\left(1 \times 10^{6}\right.$ cells/well per $1 \mathrm{ml}$ in 12 -well plates), which were obtained from the femoral tissues of wild-type mice (female, 3 months old), were cultured for $48 \mathrm{~h}$ in DMEM (10\% FBS and $1 \% \mathrm{P} / \mathrm{S})$ containing either vehicle or HCA $(1-1000 \mathrm{nM})$ in the presence or absence of the differentiation medium (DM) consisting of dexamethasone (1 $\mu \mathrm{M} / \mathrm{ml}$ medium) and IBMX $(0.5 \mu \mathrm{mM} / \mathrm{ml}$ medium; Yamaguchi et al. 2012). The medium was replaced with $\alpha$ MEM (with $10 \% \mathrm{FBS}$ and $1 \% \mathrm{P} / \mathrm{S}$ ) containing insulin $(10 \mu \mathrm{g} / \mathrm{ml}$ medium) without dexamethasone and IBMX and 
the cells were cultured in the presence of HCA $(1-1000 \mathrm{nM})$ or its absence for 4 days in a $\mathrm{CO}_{2}$ incubator $\left(37^{\circ} \mathrm{C}\right)$. In other experiments, the cells were cultured in DM for $48 \mathrm{~h}$ with HCA (1-1000 nM) or without HCA and then the medium was replaced and the cells were cultured in medium containing insulin $(10 \mu \mathrm{g} / \mathrm{ml}$ medium) for 4 days without HCA. After culture, the medium was removed and the adipocytes were stained with Oil Red O. Adipocytes were counted by light microscopy. For quantification, the dye was extracted with $0.2 \mathrm{ml}$ isopropanol for $1 \mathrm{~min}$ and the absorbance $(490 \mathrm{~nm})$ was read by using a Spectra Count microplate photometer.

\section{Culture of 3T3-L1 preadipocytes}

3T3-L1 mouse embryo fibroblasts were obtained from the American Type Culture Collection and were cultured as described elsewhere (Hemati et al. 1997). The cells were stored in liquid nitrogen and were thawed in a $37^{\circ} \mathrm{C}$ water bath on the day of culture. Cells were then maintained in a $37^{\circ} \mathrm{C} / 5 \%$ $\mathrm{CO}_{2}$ humidified environment. Briefly, 3T3-L1 preadipocytes (5000 cells/well per $0.2 \mathrm{ml}$ ) were cultured in DMEM containing $10 \%$ bovine calf serum and $1 \% \mathrm{P} / \mathrm{S}$ in $96-$ well plates in order to obtain subconfluent undifferentiated cells. After preculture (day 0 ), the undifferentiated cells were cultured in DM consisting of DMEM (with $10 \%$ FBS and $1 \% \mathrm{P} / \mathrm{S}$ ) containing $0.5 \mathrm{mM}$ IBMX and $1 \mu \mathrm{M}$ dexamethasone for 2 days and then DM was replaced with $10 \%$ DMEM containing insulin $(5 \mu \mathrm{g} / \mathrm{ml})$ with HCA $(0.1-10 \mu \mathrm{M})$ or without HCA and the cells were cultured for 2 additional days.

\section{Lipid assay}

Lipid (triglyceride) content in the cells after cell culture was quantified by using the commercially available AdipoRed assay reagent according to the manufacturer's instructions (Louza, Walkersville, Md., USA; Hemati et al. 1997; Rayalam et al. 2007). Fluorescence was measured at $485-570 \mathrm{~nm}$ in a 96 -well plate reader ( $\mu$ Quant, Bio-Tek Instruments).

\section{Cell viability assay}

Cell viability assay (CellTiter 96 Aqueous One Solution Cell Proliferation Assay; Promega, Madison, Wis., USA) was performed according to the manufacturer's instructions. Fluorescence was measured at $560-590 \mathrm{~nm}$ in a 96-well plate reader ( $\mu$ Quant, Bio-Tek Instruments) to determine the formazan concentration, which is proportional to the number of cells (Hemati et al. 1997; Rayalam et al. 2007).

Statistical analysis

Statistical significance was determined by using GraphPad InStat version 3 for Windows XP (GraphPad Software, La
Jolla, Calif., USA). Multiple comparisons were performed by one-way analysis of variance (ANOVA) with the TukeyKramer multiple comparisons post test for parametric data as indicated. $P<0.05$ was considered statistically significant.

\section{Results}

HCA stimulates osteoblastogenesis and mineralization in bone marrow culture

To determine the effects of HCA on osteoblastogenesis and mineralization, mouse bone marrow cells were cultured in medium containing either vehicle or mineralization medium in the presence of HCA $(1-1000 \mathrm{nM})$ or its absence for 21 days. Culture with HCA stimulated osteoblastogenesis and mineralization (Fig. 1a, b). Such an effect was also seen when bone marrow cells were cultured in the presence of HCA for 7 days, after which the medium was replaced with medium without HCA and the cells were cultured for an additional 14 days (Fig. 1c). Thus, HCA probably stimulated the differentiation of osteoblasts from bone mesenchymal stem cells.

HCA suppresses adipogenesis in bone marrow cells

To determine the effects of HCA on adipogenesis, mouse bone marrow cells were cultured in medium containing vehicle or in DM in the presence of HCA $(1-1000 \mathrm{nM})$ or its absence for 6 days. Culture with HCA suppressed adipogenesis (Fig. 2a, b). Such an effect was also seen when bone marrow cells were cultured in the presence of HCA for 2 days, after which the medium was replaced with the medium without HCA and the cells were cultured for an additional 4 days (Fig. 2c, d). Thus, culture with HCA suppressed adipogenesis in bone marrow cell culture.

HCA suppresses adipogenesis in 3T3-L1 preadipocytes

To determine the effect of HCA on adipogenesis in preadipocytes, mouse 3T3-L1 preadipocytes were cultured in DM for 2 days with HCA (1-100 nM) or without HCA. The medium was then replaced with DMEM containing insulin without HCA (Fig. 3a). Adipogenesis was significantly decreased (Fig. 3a). In the second experiment, preadipocytes were cultured for 2 days in DM with HCA (1-100 nM) or without HCA followed by an additional 2 days in DMEM containing insulin and HCA (1-100 nM; Fig. 3b). HCA (10 and $100 \mathrm{nM}$ ) potently suppressed adipogenesis (Fig. 3b). These results indicated that longer culture with HCA had a potent suppressive effect in adipogenesis in the presence of insulin in vitro. HCA might suppress lipid production and/or stimulate lipid degradation in adipocytes. 
a
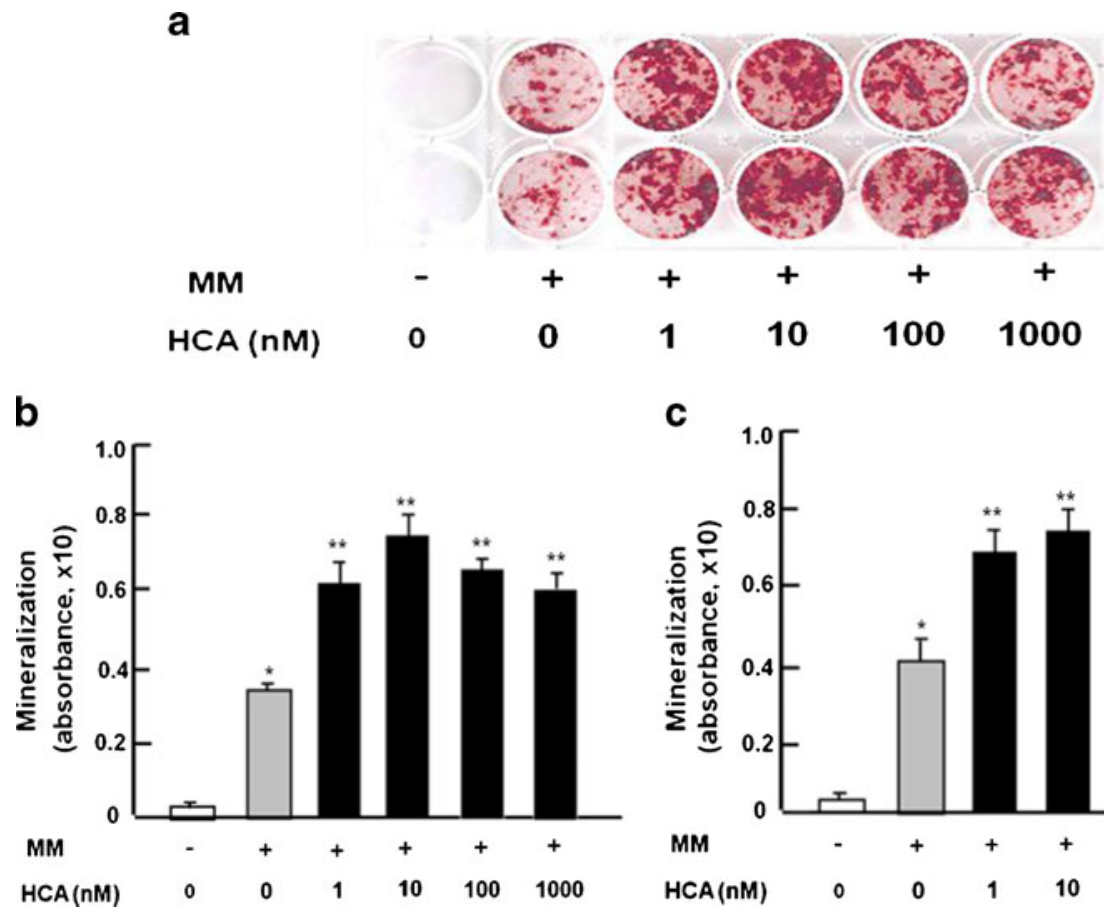

C

Fig. 1 HCA stimulates osteoblastogenesis and mineralization in mouse bone culture in vitro. Bone marrow cells were cultured in 12-well plates in DMEM (10 \% FBS and $1 \% \mathrm{P} / \mathrm{S})$ and mineralization medium $(M M)$ containing ascorbic acid $(100 \mathrm{ng} / \mathrm{ml})$ and $4 \mathrm{mM} \beta$-glycerophosphate in the presence of either vehicle or HCA (1-1000 nM). a Cells were cultured for 21 days and the medium was changed every 3 days. After culture, cells were stained with Alizarin red stain. b For quantitation, $10 \%$ cetylpyridinium chloride solution was added to each well to elute the Alizarin red dye. After complete elution, the absorbance of the eluted

HCA did not have a significant effect on cell viability. Subconfluent 3T3-L1 preadipocytes were cultured for 2 days in DM containing IBMX, dexamethasone and insulin in the presence of either the vehicle or HCA $(0.1-100 \mathrm{nM})$ or their absence and were then cultured for 2 days in medium containing insulin in the presence of either the vehicle or HCA $(0.1-100 \mathrm{nM})$ or their absence. The cell viability of mature adipocytes was not significantly changed after culture with HCA (data not shown), indicating that culture with HCA did not cause cell death.

HCA suppresses insulin-stimulated lipogenesis in mature 3T3-L1 adipocytes

To characterize the effect of HCA on the insulin-stimulated lipid accumulation, 3T3-L1 preadipocytes were cultured for 2 days in DM without HCA followed by 2 days in DMEM with HCA ( 0.1 to $100 \mathrm{nM})$ or without HCA in the presence or absence of insulin. Culture with HCA did not cause a significant change in lipid content in mature adipocytes cultured in the absence of insulin (Fig. 4). This result indicated that HCA did not have an effect on insulin-independent lipid accumulation in

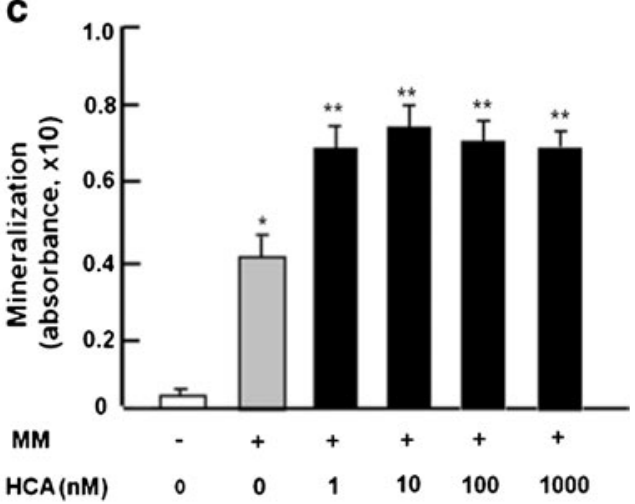

solution was measured at $570 \mathrm{~nm}$ on a microtiter plate reader. c Cells were cultured for 7 days in the presence of HCA $(1-1000 \mathrm{nM})$ or its absence and then for an additional 14 days without HCA. Quantitation was performed as in $\mathbf{b}$ ( + presence of reagents, - absence of reagents). Data are expressed as means $\pm \mathrm{SD}$ of 8 replicate samples per data set. ${ }^{*} P<0.001$ versus no treatment, ${ }^{* *} P<0.001$ versus MM-stimulated only (gray bar). One way analysis of variance (ANOVA), Tukey-Kramer post test

mature adipocytes. However, HCA (10 and $100 \mathrm{nM}$ ) had a potent suppressive effect on lipogenesis in the presence of insulin (Fig. 4). Moreover, to determine whether HCA had an effect on lipolysis, undifferentiated preadipocytes were cultured in DM without insulin addition for 2 days and differentiated mature adipocytes were cultured in medium containing HCA $(0.1-100 \mathrm{nM})$ in the absence of insulin for 2 days. Culture with HCA did not cause a significant change in lipid accumulation in adipocytes cultured in the absence of insulin (Fig. 5), suggesting that HCA did not have an effect on lipolysis in adipocytes.

Effect of signaling inhibitors on HCA-induced suppression of adipogenesis in preadipocytes cultured with insulin

To determine whether suppressive effects of HCA on insulinstimulated adipogenesis are mediated through insulin signaling, mature adipocytes were cultured in the presence of staurosporine, an inhibitor of general protein kinases including protein kinase C (Chen et al. 2009), or PD98059, an inhibitor of MAPK, which is related to the extracellularsignal-related kinase (ERK) signaling pathway (Liu et al. 

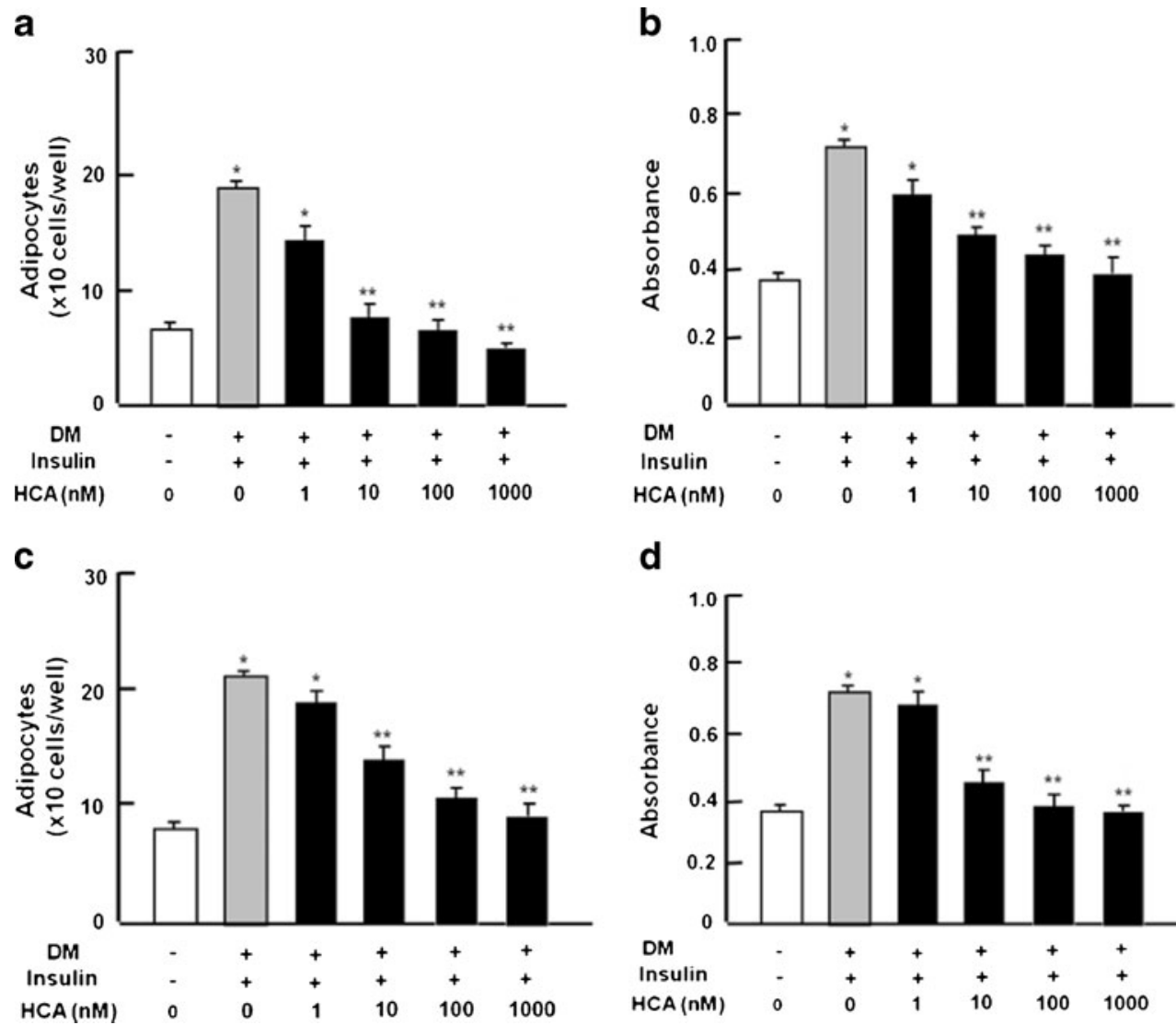

d

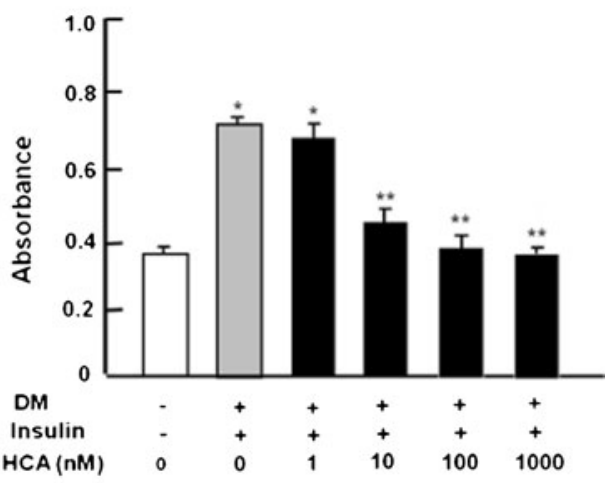

Fig. 2 HCA suppresses adipogenesis in bone marrow culture in vitro. a Bone marrow cells $\left(1 \times 10^{6}\right.$ cells/well per $1 \mathrm{ml}$ in 12 -well plates $)$ were cultured for $48 \mathrm{~h}$ in DMEM (10 \% FBS and $1 \% \mathrm{P} / \mathrm{S})$ containing either vehicle or HCA $(1-1000 \mathrm{nM})$ in the presence $(+)$ or absence $(-)$ of differentiation medium $(D M)$. The medium was replaced with $\alpha$-MEM $(10 \% \mathrm{FBS}$ and $1 \% \mathrm{P} / \mathrm{S})$ containing insulin $(10 \mu \mathrm{g} / \mathrm{ml}$ medium $)$ without dexamethasone and 3-isobutyl-1-methylxanthine (IBMX) and the cells were cultured in the presence of HCA $(1-1000 \mathrm{nM})$ or its absence for 4 days. c Bone marrow cells were cultured in DM for $48 \mathrm{~h}$ with HCA (1-

$1000 \mathrm{nM}$ ) or without HCA and then the cells were cultured in medium containing insulin $(10 \mu \mathrm{g} / \mathrm{ml}$ medium) for 4 days without HCA. After culture, the medium was removed and adipocytes were stained with Oil Red O. Adipocytes were counted by light microscopy (a, c). For quantification, the dye was extracted with isopropanol and the absorbance $(490 \mathrm{~nm})$ was read by using a Spectra Count microplate photometer (b, d). Data are expressed as means $\pm \mathrm{SD}$ of 8 replicate samples per data set. $* P<0.001$ versus no treatment; ${ }^{* *} P<0.001$ versus DM-stimulated only (gray bar). One way ANOVA, Tukey-Kramer post test
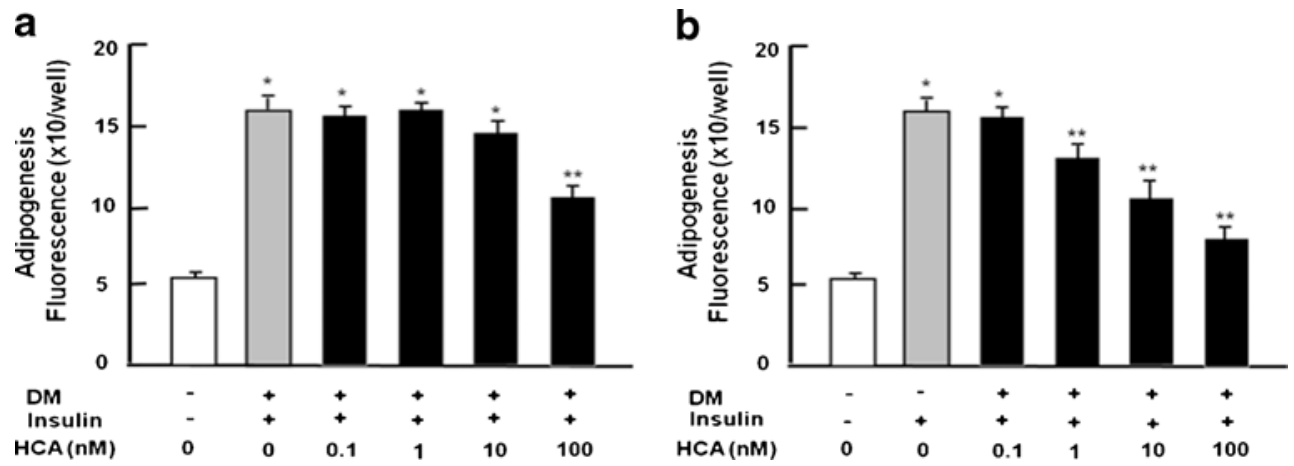

Fig. 3 HCA suppresses adipogenesis in 3T3-L1 preadipocytes. a Subconfluent preadipocytes were stimulated with differentiation medium $(D M)$ containing either vehicle or $0.5 \mathrm{mM}$ IBMX and $1 \mu \mathrm{M}$ dexamethasone for 2 days in the presence ( + ) of insulin $(5 \mu \mathrm{g} / \mathrm{ml}$ medium) without HCA. Once mature adipocytes were seen, DM was replaced with medium containing insulin $(5 \mu \mathrm{g} / \mathrm{ml})$ in the absence $(-)$ or presence $(+)$ of HCA

(1-100 nM) for 2 days (a, b). After such culture, DM was then replaced with medium containing insulin $(5 \mu \mathrm{g} / \mathrm{ml})$ without $\mathrm{HCA}$ (a) or with $\mathrm{HCA}$ (1-100 nM; b) for 2 days. After culture, lipid content was measured. Data are expressed as means $\pm \mathrm{SD}$ of 8 replicate samples per data set. ${ }^{*} P<0.001$ versus no treatment; $* * P<0.001$ versus DM-stimulated only (gray bar). One way ANOVA, Tukey-Kramer post test 


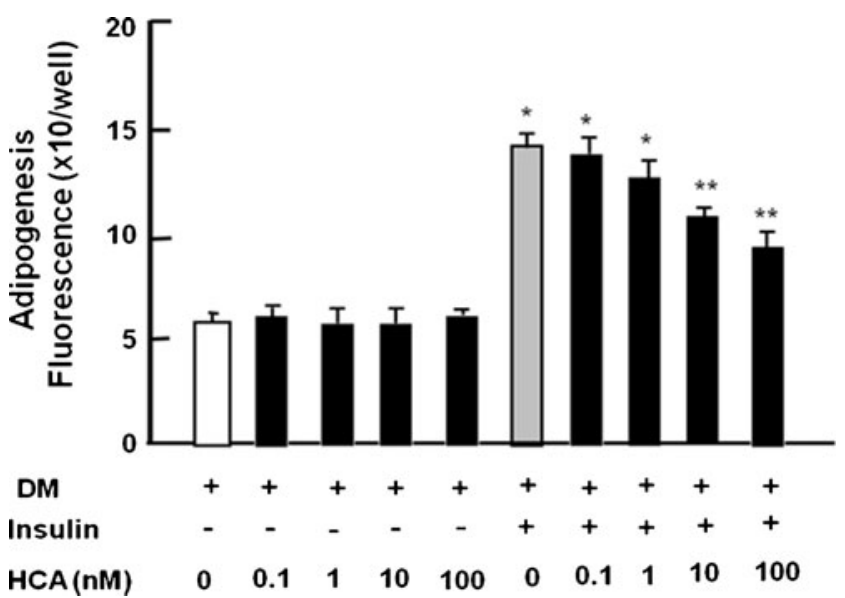

Fig. 4 HCA suppresses insulin-stimulated lipid accumulation in 3T3-L1 mature adipocytes. Subconfluent preadipocytes were cultured in differentiation medium $(D M)$ containing $0.5 \mathrm{mM}$ IBMX and $1 \mu \mathrm{M}$ dexamethasone for 2 days without HCA and then DM was replaced with medium containing either vehicle or insulin $(5 \mu \mathrm{g} / \mathrm{ml})$ in the presence $(+)$ of HCA $(0.1-100 \mathrm{nM})$ or absence $(-)$ of HCA and the cells were cultured for an additional 2 days. After culture, lipid content was measured. Data are expressed as means $\pm \mathrm{SD}$ of 8 replicate samples per data set. $* P<0.001$ versus no treatment (white bar). One way ANOVA, Tukey-Kramer post test

2012) (Fig. 6). Staurosporine or PD98059 alone did not have an effect on lipid content in adipocytes cultured without insulin. In the absence of HCA, PD98059 suppressed insulinstimulated lipid accumulation in adipocytes but no additional suppression occurred when HCA $(100 \mathrm{nM})$ was added to the medium. In the absence of HCA, staurosporine had no effect on

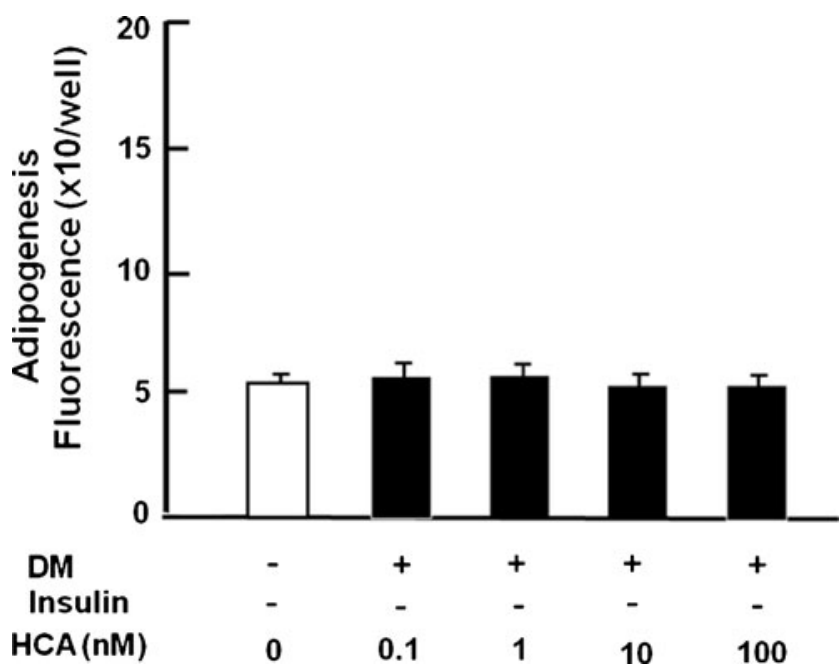

Fig. 5 HCA does not have an effect on lipid content in 3T3-L1 adipocytes cultured in the absence (-) of insulin. Subconfluent preadipocytes were cultured in differentiation medium $(D M)$ containing either vehicle or $0.5 \mathrm{mM}$ IBMX and $1 \mu \mathrm{M}$ dexamethasone for 2 days in the absence of insulin. DM was then replaced with medium containing either vehicle or HCA (0.1-100 nM) in the absence of insulin for 2 days. After culture, lipid content was measured (+ presence). Data are expressed as means \pm SD of 8 replicate samples per data set. No significant differences were seen. One way ANOVA, Tukey-Kramer post test

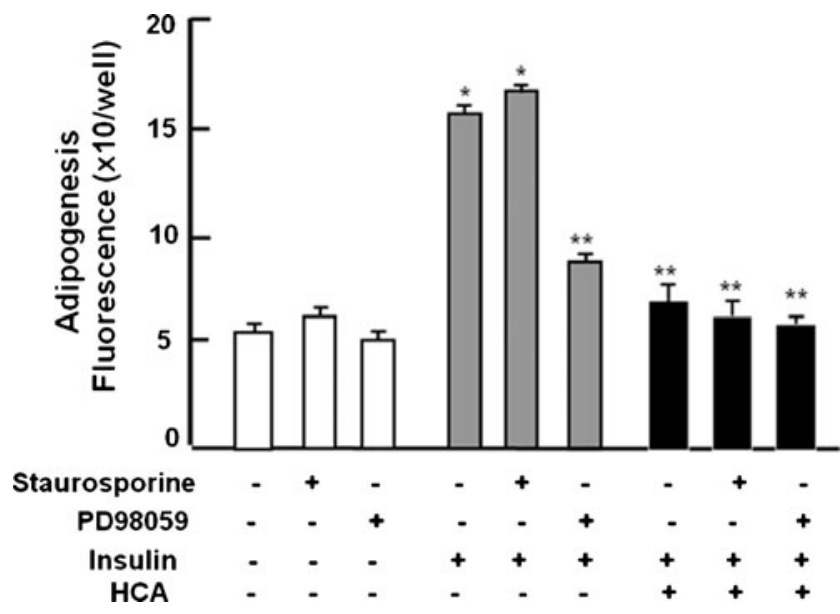

Fig. 6 Suppressive effects of HCA on adipogenesis in 3T3-L1 preadipocytes is absent in the presence of PD98059. Subconfluent preadipocytes were stimulated with differentiation medium $(D M)$ containing $0.5 \mathrm{mM}$ IBMX plus $1 \mu \mathrm{M}$ dexamethasone for 2 days in the presence $(+)$ of insulin $(5 \mu \mathrm{g} / \mathrm{ml}$ medium $)$ or absence $(-)$ of insulin with staurosporine $(0.1 \mu \mathrm{M})$, PD98059 $(1 \mu \mathrm{M})$, or HCA $(100 \mathrm{nM})$ or without these factors. DM was then replaced with medium containing either vehicle or insulin $(5 \mu \mathrm{g} / \mathrm{ml})$ with staurosporine $(0.1 \mu \mathrm{M})$, PD98059 $(1 \mu \mathrm{M})$, or HCA $(100 \mathrm{nM})$ or without these factors for 2 days. After culture, lipid content was measured. Data are expressed as means \pm SD of 8 replicate samples per data set. $* P<0.001$ versus DM-stimulated only (white bar); ${ }^{*} P<0.001$ versus insulin-stimulated only (gray bar). One way ANOVA, Tukey-Kramer post test

insulin-stimulated adipogenesis but adipogenesis was suppressed when HCA $(100 \mathrm{nM})$ was added. Thus, the suppressive effects of HCA on insulin-stimulated lipogenesis might involve the inhibition of the insulin-activated MAPK/ ERK signaling pathway in adipocytes.

\section{Discussion}

Osteoporosis and obesity are currently major health problems worldwide (Weitzmann and Pacifici 2006; Rosen and Bouxsein 2006) and one of their shared features is that osteoblasts and adipocytes differentiate from common precursor cells, namely mesenchymal stem cells, in the bone marrow. In previous experiments, HCA has been shown to stimulate osteoblastogenesis in preosteoblastic MC3T3-E1 cells (Yamaguchi et al. 2008a) and to suppress osteoclastogenesis in mouse bone marrow cells in vitro (Lai and Yamaguchi 2007a). Oral administration of HCA has been demonstrated to have an anabolic effect on bone in vivo (Lai and Yamaguchi 2006b, 2007b; Yamaguchi et al. 2008b). In this study, HCA has been found to have stimulatory effects on osteoblastogenesis and mineralization in mouse bone marrow cell culture in vitro, supporting findings that the compound has stimulatory effects on osteoblast differentiation and mineralization in preosteoclastic MC3T3-E1 cells in vitro (Yamaguchi et al. 2008a). In addition, HCA has been found to have suppressive effects on adipogenesis in mouse bone marrow 
cell culture in vitro. Osteoblasts and adipocytes differentiate from bone marrow mesenchymal stem cells (Muruganandan et al. 2009). The stimulatory effect of HCA on osteoblastogenesis and mineralization and its suppression of adipogenesis in bone marrow culture have also observed at earlier stages of culture during the differentiation of mesenchymal stem cells to preosteoblasts and preadipocytes. HCA might stimulate the process of differentiation to osteoblasts and suppress the process of differentiation of adipocytes from bone marrow mesenchymal stem cells. However, whether HCA has a direct effect on mesenchymal stem cells remains to be elucidated.

We also examined whether HCA has suppressive effects on adipogenesis during the process of differentiation from preadipocytes to mature adipocytes. Culture with HCA has been found to suppress adipogenesis in 3T3-L1 preadipocytes. HCA does not have an effect on the cell viability of adipocytes, indicating that the suppressive effects of HCA on adipogenesis do not result from cell toxicity and apoptotic cell death in preadipocytes and mature adipocytes. In addition, HCA does not stimulate lipolysis in mature adipocytes. HCA might thus suppress adipogenesis through other mechanisms but not via the stimulation of cell apoptosis and cell proliferation of adipocytes and lipolysis in mature adipocytes.

HCA suppresses adipogenesis in the differentiation process from preadipocytes to mature adipocytes in the presence of insulin. HCA might thus suppress adipogenesis during the process of differentiation from preadipocytes to mature adipocytes via a mechanism that is related to insulin action.

The activation of the MAPK/ERK signaling pathway during adipogenesis enhances the activity of factors that regulate the expression of both CCAAT/enhancer-binding protein $(\mathrm{C} /$ EBP) alpha and PPAR $\gamma$ (Prusty et al. 2002). Activation of the MAPK/ERK signaling pathway promotes PPAR $\gamma$ expression and phosphorylation and subsequently enhances the adipogenic differentiation of mesenchymal stem cells (Wu et al. 2010). The phosphorylation of Akt and ERK in 3T3L1 preadipocytes functions as downstream signaling of insulin (Wu et al. 2010; Kato et al. 2007). PD98059 is an inhibitor of the MAPK/ERK signaling pathway (Kato et al. 2007). Culture with HCA suppresses insulin-stimulated adipogenesis in 3T3-L1 preadipocytes and mature adipocytes. Such an effect is not seen in culture with PD98059, which inhibits insulin-stimulated adipogenesis in adipocytes. HCA might thus suppress insulin-stimulated adipogenesis that is mediated through the MAPK/ERK signaling pathway. We speculate that HCA directly inhibits the activity of MAPK/ERK. The molecular mechanism by which HCA suppresses insulinstimulated lipogenesis in adipocytes, however, remains to be elucidated.

In conclusion, this study demonstrates that HCA has stimulatory effects on osteoblastogenesis and mineralization and suppressive effects on adipogenesis in mouse bone marrow cell culture in vitro and that it has suppressive effects on insulin-stimulated adipogenesis in preadipocytes. Adipocyte hypertrophy and hyperplasia are important processes in the development of obesity, which induces osteoporosis. The flavonoid HCA might therefore have preventive effects on osteoporosis and obesity.

Acknowledgements M.Y. was partly supported by an Award of the Mishima Kaiun Memorial Foundation and by a Senji Miyata Foundation Award (Japan).

Open Access This article is distributed under the terms of the Creative Commons Attribution License, which permits any use, distribution and reproduction in any medium, provided the original author(s) and the source are credited.

\section{References}

Chen J-R, Lazarenko OP, Wu X, Kang J, Blackburn ML, Shankar K, Badger TM, Ronis MJJ (2010) Dietary-induced serum phenolic acids promote bone growth via $\mathrm{p} 38 \mathrm{MAPK} / \beta$-catenin canonical Wnt. J Bone Miner Res 25:2399-2411

Chen QW, Edvinsson L, Xu CB (2009) Role of ERK/MAPK in endothelin receptor signaling in human aortic smooth muscle cells. BMC Cell Biol 10:52-64

Gharibi B, Abraham AA, Ham J, Evanse BAJ (2011) Adenosin receptor subtype expression and activation influence the differentiation of mesenchymal stem cells to osteoblasts and adipocytes. J Bone Miner Res 26:2112-2124

Hemati N, Ross SE, Erickson RL, Manson MM, Walker RA, Gescher A (1997) Signaling pathways through which insulin regulates CCAAT/enhance binding protein alpha (C/EBPalpha) phosphorylation and gene expression in 3T3-L1 adipocytes. Correlation with GLUT4 gene expression. J Biol Chem 272:25913-25919

Johnell O, Kanis JA (2006) An estimate of the worldwide prevalence and disability associated with osteoporotic fractures. Osteoporos Int 17:1726-1733

Kato Y, Ozaki N, Yamada T, Miura Y, Oiso Y (2007) H-89 potentiates adipogenesis in 3T3-L1 cells by activating insulin signaling independently of protein kinase A. Life Sci 80:476-483

Kawai M, Rosen CJ (2010) Bone: adiposity and bone accrual—still an established paradigm? Nat Rev Endocrinol 6:63-64

Lai YL, Yamaguchi M (2006a) Phytocomponent $p$-hydroxycinnamic acid stimulates bone formation and inhibits bone resorption in rat femoral tissues in vitro. Mol Cell Biochem 292:45-52

Lai YL, Yamaguchi M (2006b) Oral administration of phytocomponent $p$-hydroxycinnamic acid has anabolic effects on bone calcification in femoral tissues of rats in vivo. J Health Sci 52:308-312

Lai YL, Yamaguchi M (2007a) Phytocomponent $p$-hydroxycinnamic acid inhibits osteoclast-like cell formation in mouse bone marrow cultures. Int J Mol Med 19:123-128

Lai YL, Yamaguchi M (2007b) Oral administration of phytocomponent $p$-hydroxycinnamic acid has a preventive effect on bone loss in streptozotocin-induced diabetic rats. Int J Mol Med 19:803-807

Laudes M (2011) Role of WNT signaling in the determination of human mesenchymal stem cells into preadipocytes. J Mol Endocrinol 46:R65-R72

Liu Y, Yang Y, Ye YC, Shi QF, Chai K, Tashiro S, Onodera S, Ikejima T (2012) Activation of ERK-p53 and ERK-mediated phosphorylation of Bcl-2 are involved in autophagic cell death induced by the c-Met 
inhibitor SU11274 in human lung cancer A549 cells. J Phramacol Sci 118:423-432

Minguell JJ, Erices A, Conget P (2001) Mesenchymal stem cells. Exp Biol Med 226:507-520

Muruganandan S, Roman AA, Sinal CJ (2009) Adipocyte differentiation of bone marrow-derived mesenchymal stem cells: cross talk with the osteoblastogenic program. Cell Mol Life Sci 66:236-253

Prusty D, Park BH, Davis KE, Farmer SR (2002) Activation of MEK/ ERK signaling promotes adipogenesis by enhancing peroxisome proliferator-activated receptor gamma (PPAR gamma) and C/EBP alpha gene expression during the differentiation of 3T3-L1 preadipocytes. J Biol Chem 277:46226-46232

Rayalam S, Della-Fera MA, Yang J-Y, Park HJ, Ambati S, Baile CA (2007) Resveratrol potentiates genistein's antiadipogenic and proapoptic effects in 3T3-L1 adipocytes. J Nutr 137:2668-2673

Rosen CJ, Bouxsein ML (2006) Mechanisms of disease: is osteoporosis the obesity of bone? Nat Clin Pract Rheum 2:35-43

Weitzmann MN, Pacifici R (2006) Estrogen deficiency and bone loss: an inflammatory tale. J Clin Invest 116:1186-1194
Wu L, Cai X, Dong H, Jing W, Huang Y, Yang X, Wu Y, Lin Y (2010) Serum regulates adipogenesis of mesenchymal stem cells via MEK/ ERK-dependent PPARgamma expression and phosphorylation. J Cell Mol Med 14:922-932

Yamaguchi M, Weitzmann MN (2009) The bone anabolic carotenoids $p$-hydroxycinnamic acid and $\beta$-cryptoxanthine NF- $\mathrm{kB}$ activation in MC3T3 preosteoblasts. Mol Med Reports 2:641-644

Yamaguchi M, Weitzmann MN (2012) The bone anabolic carotenoid $p$-hydroxycinnamic acid promotes osteoblast mineralization and suppresses osteoclast differentiation by antagonizing NF- $\mathrm{kB}$ activation. Int $\mathrm{J}$ Mol Med 30:708-712

Yamaguchi M, Lai YL, Uchiyama S, Nakagawa T (2008a) Phytocomponent $p$-hydroxycinnamic acid stimulates mineralization in osteoblastic MC3T3-E1 cells. Int J Mol Med 22:287-291

Yamaguchi M, Lai YL, Uchiyama S, Nakagawa T (2008b) Oral administration of phytocomponent $p$-hydroxycinnamic acid prevents bone loss in ovariectomized rats. Mol Cell Biochem 311:31-36

Yamaguchi M, Weitzmann MN, Baile CA, Murata T (2012) Exogenous regucalcin suppresses osteoblastogenesis and adipogenesis in mouse bone marrow culture. Integr Biol 4:1215-1222 